\title{
Convalescent Plasma: A Potential Treatment for COVID-19
}

\author{
MAKHAN $^{\mathrm{a}}$, AMAFRUHA ${ }^{\mathrm{b}}$
}

\begin{abstract}
Summary:
As no specific standard therapies have been approved for Coronavirus disease 2019 (COVID-19), so prevention and supportive care dominate the approach to COVID-19. Exposure to this severe acute respiratory syndrome coronavirus 2 (SARS-CoV-2) results in an adaptive immune response that commonly include antibodies with neutralization activity. Treatments directly targeting the virus and the inflammatory response to it remain investigational. Convalescent plasma (CP) is such a therapy that had been reported hundred years back in studies from the Spanish influenza era. So the idea of convalescent plasma from subjects who have recovered from viral infections has been used to both prevent or treat disease. Over the past two decades' notable examples of the successful use of convalescent plasma (CP) include influenza, measles, Middle East respiratory syndrome (MERS), Ebola and severe acute respiratory syndrome (SARS). Two case series were recently published
\end{abstract}

Introduction:

December 2019, in Wuhan, China experienced a highly infectious new viral pneumonia outbreak associated with severe acute respiratory syndrome. ${ }^{1,2}$ The virus was named as severe acute respiratory syndrome coronavirus 2 (SARS-CoV-2) or in short coronavirus disease 2019 (COVID-19) by World Health Organization (WHO) ${ }^{3,4}$. The epidemic spread rapidly worldwide within 3 months and was declared as a pandemic by WHO on March 11,2020.5

COVID-19 is associated with a broad spectrum of clinical syndromes, ranging from asymptomatic or mild upper airway symptoms to progressive life threatening viral pneumonia leading to respiratory failure and death., ${ }^{2,6}$ Symptoms may develop 2 days to 2 weeks following

a. Dr. Mohiuddin Ahmed Khan, Professor of Hematology \& Bone Marrow Transplantation, Department of Hematology, Dhaka Medical College \& Hospital, Dhaka, Bangladesh.

b. Dr. Mafruha Akter, Asstt. Professor of Hematology \& Bone Marrow Transplantation, Department of Hematology, Dhaka Medical College \& Hospital, Dhaka, Bangladesh.

Address of Correspondence: Dr. Mohiuddin Ahmed Khan, Nisorgo B/7, House \# 21, Road \# 3, Dhanmondi R/A, Dhaka1205, Dhaka, Bangladesh. E-mail: dr.khan59@gmail.com by China examining the therapeutic use of CP in patients with COVID-19. In the context of pandemic situation, the Food and Drug Administration (FDA) allowed to use COVID-19 convalescent plasma as Investigational New Drug (IND) since April 2020 to help patients with serious or immediately life-threatening illness associated with COVID19. Case series studying convalescent plasma use in the treatment of COVID-19 have been promising, but additional, high-quality studies are needed to determine the efficacy of the treatment when applied for prophylaxis, for early phases of illness and for severe illness. Bangladesh also started program to use convalescent plasma for severe and critical COVID-19 patients under limited clinical trial.

Keywords: COVID-19, Convalescent plasma, eIND, NAbT, SARS-CoV-2

(J Bangladesh Coll Phys Surg 2020; 38: 109-115) DOI: https://doi.org/10.3329/jbcps.v38i0.47350

exposure to the infected person which is diagnosed by real time Reverse Transcription-Polymerase Chain Reaction (RT-PCR). Common symptoms are fever, dry cough, myalgia, fatigue, sore throat and less common presentation are headache, diarrhea, dyspnea and respiratory distress. According to CDC (Center for disease control and prevention) elder adult and persons who have underlying comorbidities such as heart disease, diabetes, lung disease and obesity are at high risk for developing severe disease. Recently it is reported that severe form of COVID-19 is also associated with coagulopathy and endothelialitis. 7,8 About 20\% patients of COVID-19 are asymptomatic and $60 \%$ are with mild to moderate symptoms may improve with home treatment. About $20 \%$ patients need admission to hospital with severe disease and around 5\% of them with critically ill need transfer to ICU. About 2 to $5 \%$ death are due to development of ARDS followed by acute respiratory failure as well coagulopathy and multiorgans involvement. CT scan of chest shows peripheral lung ground-glass opacities, the early phase of ARDS and histologically diffuse alveolar damage with oedema, hemorrhage and intra-alveolar fibrin deposition. ${ }^{8}$ 


\section{Pathophysiology:}

SARS-CoV-2 virus after entering to respiratory tract, receptor binding domain(RBD) binds to ACE2 receptor of mucous membrane. With a rapid viral replication and massive infiltration of inflammatory cells (macrophage, lymphocytes and dendric cells ) resulting elevation of pro-inflammatory cytokines and chemokines ( IL-1,IL-4, IL-6, IL-10, IL-12, IL-17, TNF-á, IFN-ã, GM-CSF etc).${ }^{9} \mathrm{~A}$ drastic elevation of inflammatory cytokines \& chemokines known as cytokine storm causes acute pulmonary injury and ultimately develop acute respiratory distress syndrome(ARDS). Increase of cytokines, LDH, CRP, lymphopenia are directly related to severity of inflammation and viral load. The reduction of CD4+ and CD8+T cells is commonly associated with lymphopenia. ${ }^{9}$ The SARS-CoV-2 S protein is likely important target for developing neutralizing antibodies (NAbs) to block binding and fusion of SARS-CoV-2 (Figure-1). SARS-CoV-2 seems to use the same cell entry receptor, ACE2, as the SARS-CoV because ACE2 shows binding to RBD of both SARS-CoV and SARS-CoV-2. Understanding of SARS-CoV-2 immuno-pathogenesis is useful for developing passive antibody therapy, designing vaccines and understanding of clinical drug interventions. The systemic landscape of the immune responses in patients with COVID-19 is unclear. But clinical features and immunopathogenesis of SARS-
CoV-2 have many similarities with SARS-CoV-1. The development of immunity to a pathogen through natural infection is a multi-steps process that typically takes place 1-2 weeks. The body responds to a viral infection immediately with a non-specific innate response in which macrophages, neutrophils, dendritic cells slow the progress of virus and may even prevent it from causing symptoms. ${ }^{10}$ This non-specific response is followed by an adaptive response where the body makes antibodies that specifically bind to the virus. These antibodies are proteins called immunoglobulin. The body also makes T-cells that recognize and eliminate other cells infected with the virus, called cellular immunity. These combined adaptive responses may clear the virus from the body, and if the response is strong enough, may prevent progression to severe illness ${ }^{11}$. This process is often measured by the presence of antibodies in the blood.

Also there is an evidence of microvascular thrombosis in the lungs, known as COVID associated coagulopathy $(\mathrm{CAC})$ resulting from endothelial (vascular $\&$ myocardial) injury and activation of coagulation cascades, ultimately thrombotic microangiopathy and eventually multi-organs failure often leading to death. ${ }^{12}$

\section{Historical precedents and Scientific rationale of CP use:}

Passive antibody therapy was first described in the 1890 s prior to the development of antimicrobial therapy. ${ }^{11}$
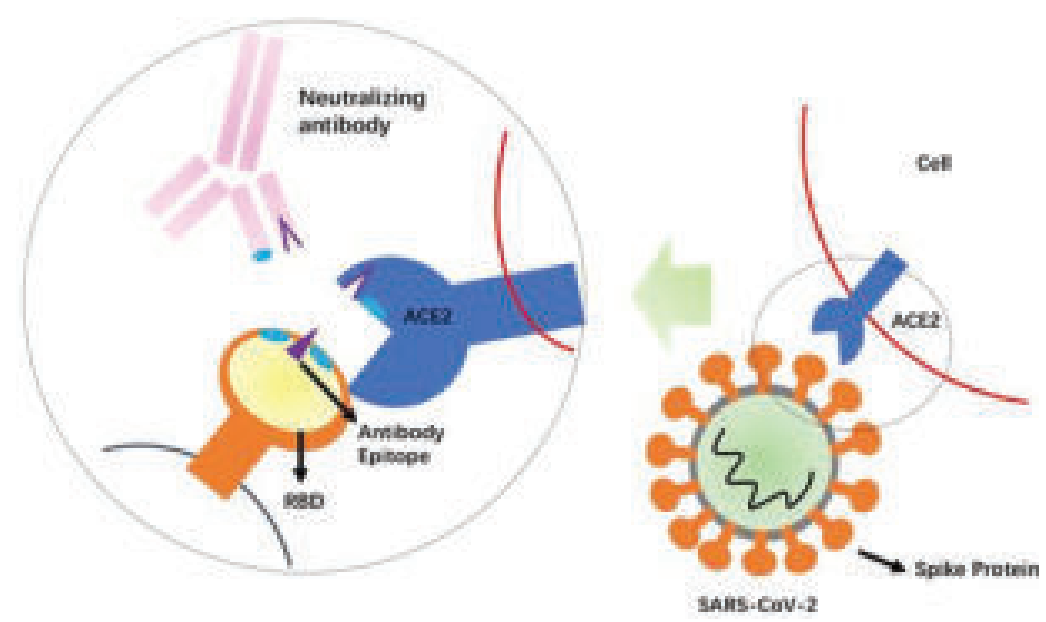

Fig-1: Schematic mechanism of the Neutralizing antibodies. Competition of NAb with ACE2 receptor for binding to the receptor-binding domain(RBD) of SARS-CoV-2 Spike protein is shown. The protruding portion(violet) of $R B D$ is both the ACE2 receptor-binding site and the antibody epitope. ${ }^{9}$ 
Convalescent plasma was used during the 1918 Spanish flu epidemic and reduced mortality among plasma recipients. ${ }^{13}$ More recently, severe acute respiratory syndrome coronavirus 1 (SARS-CoV-1) in 2003 and Middle East respiratory syndrome (MERS) in 2012 both viral outbreaks with high mortality and absence of effective therapies led to the use of convalescent plasma. ${ }^{14,15}$ In the largest study of the SARS-CoV-1 outbreak, among 80 patients in Hong Kong, patients treated within the first 14 days of infection had earlier discharge from hospital. ${ }^{16}$ These results are consistent with that convalescent plasma may be an effective treatment of coronavirus infections and that earlier administration is more likely to be successful. ${ }^{17}$

Human convalescent plasma may be an option for treatment COVID-19 as no specific treatment is approved till date and could be rapidly available where there are sufficient numbers of people recovered can donate plasma without any harm. This passive immunity (antibody) therapy can be given to a susceptible individual for preventing and treating an infectious disease such as COVID-19. In contrast, active immunity requires induction of immune response (e.g. by vaccination) that requires time to develop and moreover in some immuno-compromised patients fail to achieve adequate immune response. Thus passive antibody administration is the only means of providing immediate immunity to susceptible persons and immunocompromised patients. Experience from prior out breaks of H1N1 influenza (Spanish virus), Ebola, SARS-1 and MERS virus; where convalescent plasma was also used prior to developed specific treatment. There are few reports that convalescent plasma was used for therapy for critically ill patients with COVID-19 in China during the current outbreak. ${ }^{17,18}$ The effectiveness of CP therapy was also evaluated on 10 COVID-19 patients including 3 were in ventilation; clinical symptoms of them were significantly improved along with increased of oxyhemoglobin saturation within 3 days, several parameters tended to improve as compared to pretransfusion, including increased of lymphocytes count and decreased C-reactive protein, resolution of lung lesions on radiology and viral load was undetectable by 7 days after transfusion of $\mathrm{CP}^{17}$. In another small case series, five critically ill of COVID-19 patients with ARDS were treated with convalescent plasma containing neutralizing antibodies. Infusion of plasma was followed by improvement in clinical status in all trial patients, with no deaths. ${ }^{18}$ It was observed that patients who received convalescent plasma in early phase of illness had better outcome and early hospital discharge. So when used for therapy, antibodies are most effective if administered shortly after the onset of symptoms. The reason is that initially virus is likely to be much smaller in amount than that of established severe disease. Another explanation is that passive antibody therapy works by modifying the inflammatory response, which is also easier during the initial phase ${ }^{19}$. Depending on the antibody amount and composition, the protection given by transfused immunoglobulin varies and last for weeks to months.

In response to the COVID-19 outbreak in the US and reported high case-fatality rates, the US Food and Drug Administration (FDA) on $3^{\text {rd }}$ April has approved the use of COVID-19 convalescent plasma (CCP) from recovered patients to treat people as emergency investigational new drug(eIND) who are severe and critically ill with COVID-19. ${ }^{20}$ Worldwide in sixty countries including USA and UK are running national clinical trials on convalescent plasma therapy to gather evidence across the global disease spectrum.

\section{COVID-19 Convalescent Plasma (CCP):}

Convalescent plasma is the liquid part of blood that is removed from the blood of a person who has recovered from a disease containing particular antibody. Plasma collected from COVID-19 recovered patient is known as COVID-19 convalescent plasma (CCP) that can be used for other patients to boost up passive immunity. Specific drugs or vaccine are not yet developed, so about $15 \%$ $20 \%$ patients who are deteriorating only depend on supporting management. Among these patients who are deteriorating (increasing respiratory distress and decreasing arterial O2 saturation) COVID-19 convalescent plasma could be given as emergency investigational treatment. Convalescent plasma has been used successfully for the treatment of a variety of infectious disease such as H1N1, Ebola, SARS-CoV-1, MERS virus. Outcome is better when CP given in the early phase of the infection than late phase when disease is established.

\section{Donor eligibility: ${ }^{21}$}

COVID-19 convalescent plasma must only be collected from individuals $\geq 18$ years who meet all donor eligibility 
requirements and in addition who meet the following qualifications: ${ }^{21}$

- Evidence of COVID-19 documented by a laboratory test either by a diagnostic test (e.g., nasopharyngeal swab) at the time of illness or a positive serological test for SARS-CoV-2 antibodies after recovery, if prior diagnostic testing was not performed at the time COVID-19 was suspected.

- Complete resolution of symptoms at least 14 days prior to donation. A negative result for COVID-19 by a diagnostic test is not necessary to qualify the donor.

- Screening tests for safe blood transfusion like HBsAg, anti-HCV, anti-HIV, VDRL and malaria must be negative.

- Male donors, or female donors who have not been pregnant, or female donors who have been tested since their most recent pregnancy and results interpreted as negative for HLA antibodies.

- SARS-CoV-2 neutralizing antibody titer, if available (titer of at least 1:160). When measurement of neutralizing antibody titer is not available, consider storing a retention sample from the convalescent plasma donation for determining antibody titer at a later date

An eligible COVID-19 donors can donate plasma repeatedly at 2-4 weeks interval.

\section{Collection, Processing and storage:}

Donor will ideally donate plasma by plasmapheresis, usually $400 \mathrm{ml}$ to $600 \mathrm{ml}(10 \mathrm{ml} / \mathrm{kg})$ plasma can be collected by plasmapheresis and should be split before freezing into 2 -3 separate units each with $200 \mathrm{ml}$. Final products should be specifically labelled as COVID-19 convalescent plasma and should be Fresh Frozen, preferably should be frozen within 8 hours after collection, stored at $-18^{0} \mathrm{C}$ or colder and have an expiration date one year from the date of collection. Convalescent plasma may be stored at $1-6^{0} \mathrm{C}$ up to 40 days. ${ }^{21,22}$

Although plasmapheresis is the standard procedure for collection of plasma but in some places particularly in low and middle income countries, where plasmapheresis facilities are not widely available, whole blood can also be collected with plasma component separation by centrifugation technique following standard operating procedures. $^{21}$

\section{Testing antibody titer of donated plasma:}

People who have recovered from SARS-Cov-2 infection have antibodies to the virus. However, some of these people have very low levels of antibodies in their blood, suggesting that cellular immunity may also be critical for recovery. It is strongly recommended that SARSCoV-2 neutralizing antibody titer(NAbT) be measured in the donated plasma. Current data suggest that donors with a minimal titer by end-point dilution of 1:80 or preferably 1:160 should be selected. ${ }^{21}$ When the measured neutralizing antibodies in the collected plasma is considered to be too low, the plasma should be made available for other use(ideally fractionation or FFP). In the absence of neutralizing antibody testing, a test for the presence of anti-SARS-CoV-2 antibody should ideally be performed prior to release. In emergency cases, where plasma is released for transfusion without any antibody testing, archived samples should be tested at a later date once testing is available. ${ }^{21}$ If an adequate correlation between neutralizing activity and ELISA antibody testing were to be demonstrated, this could replace the test for neutralizing antibodies. It is advised that additional archive samples of the donated plasma are saved for reference studies, e.g. 10x0.5ml frozen aliquots from plasma samples taken at the time of donation. Donor plasma samples obtained at the time of donation should be saved frozen at (minus) $-18^{0} \mathrm{C}$ or colder for retrospective testing of the total and neutralizing titers of anti-SARS-CoV-2 antibodies and further scientific investigations.

\section{Indication of use CCP:}

Importance of use of CCP is on interim period before invention of any specific drugs and vaccine. Historical evidence and small pilot studies on Convalescent plasma in COVID -19, proved that it has benefits to combat symptoms and mortality. Appropriate use of CCP could limit the transfer of COVID-19 patients to ICU.

Indication of use of CCP in laboratory confirmed COVID19 patients with severe or immediately life-threatening COVID-19 as defined with one or more of the following: ${ }^{22}$

- shortness of breath (dyspnea),

- respiratory frequency $\geq 30 / \mathrm{min}$,

- blood oxygen saturation $\leq 93 \%$,

- partial pressure of arterial oxygen to fraction of inspired oxygen ratio $<300$,

- lung infiltrates $>50 \%$ within 24 to 48 hours 
Life-threatening disease is defined as one or more of the following: ${ }^{22}$

- respiratory failure,

- septic shock,

- multiple organs dysfunction or failure

Informed consent provided by the patient or attendant.

For Prophylaxis use of CP for high risk group such as health workers who are working in COVID units and elderly person with comorbidities are still under investigation.

In addition to clinical trial to fulfil the huge demand, CP should be used as a nationwide expanded access program in a coordinated way to facilitate the only available passive antibody therapy for severe COVID19 patients.

\section{Convalescent Plasma Transfusion:}

There should be standard hospital procedures and recommendations for thawing and transfusion of plasma same as FFP and expiry 24 hours post thaw. It is crucial to ensure $\mathrm{ABO}$ compatibility of plasma between the donor and the recipient. Patient should receive an initial dose of ABO compatible 200-300 ml convalescent plasma, followed by one or two additional doses of $200 \mathrm{ml}$ according to disease severity, response and tolerance of the infusions and finally with compassionate clinical judgment. Patient should be closely monitored during and after transfusion for safety and efficacy profile of convalescent plasma. Patients should be followed up for safety and effectiveness after transfusion of convalescent plasma; like any adverse effect, improvement of $\mathrm{SpO}_{2}$, lymphopenia, CRP, liver enzymes, serum ferritin level, radiological findings, viral load as well clinical wellbeing and respiratory distress etc.

\section{Potential risks of convalescent plasma:}

Fortunately, majority of the adverse effects due to plasma transfusion are not lethal and are adequately treated in clinical practice. The incidence of allergic transfusion reactions(ATR) have been estimated as less than $1 \%$ to $3 \%$ of all transfusions ${ }^{23}$ and most ATRs are mild and limited to urticaria, purities, and/or flushing. The most dangerous and highest risk of mortality following plasma transfusion is likely due to sequelae of pulmonary complications notably TRALI and TACO known as severe adverse effects. Transfusion-related acute lung injury (TRALI) and transfusion-associated circulatory overload (TACO) are the two leading causes of transfusion-related mortality, and are often difficult to distinguish and these conditions have been emphasized in the plasma transfusion literature. The reported incidence of TACO and TRALI similarly covers a large range from $\sim 0.01 \%$ in surveillance surveys to $8 \%$, depending on the clinical status of the transfusion recipient. ${ }^{24,25}$ Exclusion of female donor who have history of pregnancy with possibility of presence of antibodies to HLA or granulocyte antigens and testing for these antibodies where feasible, lower the occurrence of TRALI. ${ }^{21}$

In a recent preprint article (before formal publication) it has been stated that after transfusion of COVID-19 convalescent plasma in 5,000 patients in US, the early incidence of adverse events was very low $(<1 \%)$ with incidence of TRALI and TACO is $<0.1 \% .{ }^{26}$

A theoretical concern of the use of COVID-19 convalescent plasma in patients with COVID-19 is a deteriorated clinical condition after plasma transfusion secondary to antibody-dependent enhancement (ADE) of infection or antibody-mediated pro-inflammatory effects. ${ }^{27}$ There is also the concern that antibody administration to individuals with significant viral loads may lead to the formation of antigen-antibody immune complexes, which may contribute to proinflammatory immune responses. ${ }^{28}$ But these are remote theoretical complications not reported with COVID-19 CP so far.

The World Health Organization (WHO) guidelines recommended convalescent plasma for use against Ebola virus disease (EVD) during Ebola outbreak in $2014 .{ }^{29} \mathrm{In}$ each of affected countries, several nonrandomized comparative clinical trials were initiated. The Ebola treatment with CP trial in 2015, no severe adverse reactions were noted. ${ }^{30}$

\section{Conclusion:}

Beyond supportive care there are no proven standard and specific treatment have been approved for COVID19 infection except clinical trial. Given the deadly nature of COVID-19 and the large population of critically-ill patients who are fighting with death, the emergency investigational treatment approach with passive immunity through convalescent plasma may not do harm to those patients. Globally $\mathrm{CP}$ is under active investigation not only as treatment for COVID-19 but 
also as prophylaxis. Because $\mathrm{CP}$ use for SARS-CoV-2 and other viruses has shown promise and administration of $\mathrm{CP}$ is not associated with lethal complication, life threatening adverse reaction risk is $<0.1 \%$. Critical factors to be considered in designing treatment protocols including timing of transfusion (early the better), clinical status of the recipient and antibody titer of the donor. As emerging data are encouraging in favour of safely and efficacy, clearly, the use of convalescent plasma may improve the clinical outcomes in patients with COVID-19 infection and could be a stopgap of current pandemic. As a middle income country we have limited number of ICU beds \& ventilators, using CP we can limit the pressure on ICU. A Coordinated planning and activities of all concerned physicians, infectious disease specialists, hematologists, transfusion medicine and DGHS can combat the COVID-19 crisis. For this a national expanded access program is needed to organize and monitoring the rational use and documentation of convalescent plasma.

\section{References:}

1. P. Zhou et al., A pneumonia outbreak associated with a new coronavirus of probable bat origin. Nature 2020; 579: 270-273.

2. N. Chen et al. Epidemiological and clinical characteristics of 99 cases of 2019 novel coronavirus pneumonia in Wuhan, China: A descriptive study. Lancet 2020; 395: 507-513.

3. World Health Organization, Coronavirus disease (COVID19) Pandemic. https://www.who.int/emergencies/ diseases/ novel-coronavirus-2019. Accessed 11 March 2020.

4. World Health Organization (WHO). Report of the WHO China Joint Mission on coronavirus disease 2019 (COVID19); February 2020. Available at www.who.int/docs/ defaultsource/ coronavirus/who-china-joint-mission-on-covid-19final-report.

5. World Health Organization (WHO). Rolling updates on coronavirus diseases (COVID-19). www.who.int/ emergencies/diseases/novel-coronavirus-2019/events-asthey-happen (accessed 13 April 2020)

6. Zhu N, Zhang D, Wang W, et al. A novel coronavirus from patients with pneumonia in China, 2019. N Engl J Med 2020; 382: 727-33.

7. Thachil J, Wada H, Gando S, et al. ISTH interim guidance on recognition and management of coagulopathy in COVID-19. J Thromb Haemost 25 March,2020; published online. DOI:10.1111/jth.14810.

8. Ackermann M, Verleden SE, Kuehnel M, et al. Pulmonary vascular endothelialitis, thrombosis, and angiogenesis in
Covid-19. N Engl J Med 2020. DOI: 10.1056/ NEJMoa2015432.

9. Zhou G, Zhao Q. Perspectives on therapeutic neutralizing antibodies against the Novel Coronavirus SARS-CoV-2. Int J Biol Sci. 2020;16(10):1718 1723. Published 2020 Mar 15. doi:10.7150/ijbs.45123.

10. Casadevall A, Pirofski L. Antibody-mediated regulation of cellular immunity and the inflammatory response. TRENDS in Immunology. 2003;24(9):474-478.

11. Casadevall A. Scharff MD. Serum therapy revised: animal models of infection and development of passive antibody therapy. Antimicrobial agents and chemotherapy. 1994;38(8): 1695 .

12. Liu Y, Yan LM, Wan L, Xian TX, Le A, Liu JM, et al. Viral dynamics in mild and severe cases of COVID-19. Lancet Infection Dis. 2020 Mar 19.

13. Luke TC, Kilbane EM, Jackson JL, and Hoffman SL. Metaanalysis: convalescent blood products for Spanish influenza pneumonia: a future H5N1 treatment? Ann Intern Med. 2006;145(8):599-609.

14. Arabi YM, Hajeer AH, Luke T, et al. Feasibility of using convalescent plasma immunotherapy for MERS-CoV infection, Saudi Arabia. Emerg Infect Dis 2016; 22: 1554-61.

15. Arabi Y, Balkhy H, Hajeer AH, et al. Feasibility, safety, clinical, and laboratory effects of convalescent plasma therapy for patients with Middle East respiratory syndrome coronavirus infection: a study protocol. Springer plus 2015; 4: 709 .

16. Ko JH, Seok H, Cho SY, et al. Challenges of convalescent plasma infusion therapy in Middle East respiratory coronavirus infection: a single centre experience. Antivir Ther 2018; 23: 617-22.

17. Duan K, Liu B, Li C, Zhang H, Yu T, Qu J, et al. Effectiveness of convalescent plasma therapy in severe COVID-19 patients. Proceedings of the National Academy of Sciences of the United States of America. 2020.

18. Shen C, Wang Z, Zhao F, Yang Y, Li J, Yuan J, et al. Treatment of 5 Critically Ill Patients With COVID-19 With Convalescent Plasma. Jama. 2020.

19. Long Chen, Jing Xing, Lei Bao et al. Convalescent plasma as apotential therapy for COVID-19. the lancet April 2020. Doi.org/10.1016/S1473-3099(20)30141-9.

20. Janice Hopkins Tanne. COVID-19: FDA approves use of Convalescent plasma to treat critically ill patients. BMJ 2020;368:m1256 doi: 10.1136/bmj.m1256 (published 26 March 2020).

21. Organizing Committee of the international society of blood transfusion(ISBT) working party on Global Blood safety. Points to consider in the preparation and transfusion of COVID-19 convalescent plasma in low and middle income countries. April 2020. 
22. U.S. Food and Drug Administration. Recommendations for Investigational COVID-19Convalescent Plasma. 2020. https://www.fda.gov/vaccines-blood-biologics/ investigational-newdrug-ind-or-device-exemption-ideprocess-cber/recommendations -investigational-covid-19convalescent-plasma-Patient 20 Eligibility,2020.

23. Vamvakas E. Allergic and anaphylactic reactions. In: Popovsky M, editor. 3rd ed. Transfusion reactions. Bethesda (MD): American Association of Blood Banks; 2007. p. 105- 56 .

24. Bosboom JJ, Klanderman RB, Migdady Y, Bolhuis B, Veelo DP, Geerts BF, et al. Transfusion-Associated Circulatory Overload: A Clinical Perspective. Transfus Med Rev. 2019;33(2):69-77.

25. Vlaar APJ, Toy P, Fung M, Looney MR, Juffermans NP, Bux J, et al. A consensus redefinition of transfusion-related acute lung injury. Transfusion. 2019;59(7):2465-76.

26. Early Safety Indicators of COVID-19 Convalescent Plasma in 5,000 Patientsdoi: https://doi.org/10.1101/ 2020.05.12.20099879 medRxiv preprint
27. de Alwis R, Chen S, Gan ES, and Ooi EE. Impact of immune enhancement on Covid-19 polyclonal hyperimmune globulin therapy and vaccine development. EBioMedicine. 2020;55:102768

28. Lutz HU. How immune complexes from certain IgG NAbs and any $F\left(a^{\prime}\right)(2)$ can mediate excessive complement activation. Adv Exp Med Biol. 2012;750:186-96.

29. World Health Organization. Use of convalescent whole blood or plasma collected from patients recovered from Ebola virus disease for transfusion, as an empirical treatment during outbreaks. Interim guidance for national health authorities and blood transfusion services. Geneva, Switzerland: WHO, 2014.

30. Van Griensven J, De Weiggheleire A, Delamou A, et al. The Use of Ebola Convalescent Plasma to Treat Ebola Virus Disease in Resource-Constrained Settings: A Perspective from the Field. Clin Infect Dis. 2016;62(1):69 74. doi: $10.1093 / \mathrm{cid} / \operatorname{civ} 680$ 